\title{
Analyzing the relationships between learners' motivation and observable engaged behaviors in a gamified learning environment
}

\author{
Élise Lavouéa,c, ${ }^{\mathrm{a}, *}$, Qinjie Ju ${ }^{\mathrm{b}, \mathrm{c}}$, Stuart Hallifax ${ }^{\mathrm{a}, \mathrm{c}}$, Audrey Serna ${ }^{\mathrm{b}, \mathrm{c}}$ \\ ${ }^{a}$ University of Lyon, Université Jean Moulin Lyon 3, iaelyon school of Management \\ ${ }^{b}$ INSA de Lyon \\ ${ }^{c}$ CNRS, LIRIS UMR5205, F-69621 Lyon, France
}

\begin{abstract}
Over the last ten years, gamification has been widely integrated in digital learning environments as a way to increase learners' motivation. However, little is known about engaged behaviors adopted by learners when using gamified learning environments. In this paper, we analyze learners' interactions with a gamified learning environment to study learners' engagement in this particular context and to identify the factors that influence engaged behaviors. We also analyze the complex relationships that exist between learners' engagement and motivation. We conducted a large-scale field study in ecological conditions, involving 257 students (13-14 years' old) in 12 classes, from 4 different middle schools. We identified a model of engagement that distinguishes two types of engaged behaviors: an achievement-oriented engagement for initially intrinsically motivated learners or high achiever learners, and a perfection-oriented engagement for low achiever learners. We show that each type of engaged behavior has a specific impact on the variation in learners' motivation during the learning activity. This model contributes to a better understanding of how gamification can affect learners' engaged behaviors and motivation during the learning activity according to their initial motivation and player profile. These findings open up new perspectives in
\end{abstract}

\footnotetext{
*Corresponding author

Email addresses: elise.lavoue@liris.cnrs.fr (Élise Lavoué), qinjie.ju@liris.cnrs.fr (Qinjie Ju), stuart.hallifax@liris.cnrs.fr (Stuart Hallifax), audrey.serna@liris.cnrs.fr (Audrey Serna)
} 
terms of motivational affordances, as well as the design and dynamic adaptation of gamification based on learners' interaction traces with the learning environment.

Keywords: Gamification, Digital learning environment, Behavior analysis, Motivation, Engagement, Player profile

\section{Introduction}

Over the last ten years, gamification has been widely integrated in learning environments as a way to increase learners' motivation. This technique relies on the use of game design elements in non-game contexts [1]. Although many studies have been dedicated to the evaluation of the impact of gamification on learners' motivation, little is known about its impact on learner engagement. While the concept of engagement has been thoroughly studied and defined in the literature for several decades $[2,3,4]$, in recent years it has generated a great deal of interest in the field of digital education $[5,6,7]$. This is the case for online learning (e.g. MOOCs) for example, where the percentage of dropouts is high, and where keeping learners engaged in a course is a major challenge for educational institutions [8].

In this paper, we focus on observable engaged behaviors through learners' interactions with the learning environment. We address the overarching questions: what is the impact of a gamified learning environment on learners' engaged behaviors, and what are the factors that influence these behaviors? Today, it is fully acknowledged that the motivational impact of gamification depends on individual factors, such as player profile $[9,10,11,12]$ and learners' motivation for the learning task $[13,14]$. However, no study has yet been conducted on the influence of these individual factors on the different types of engaged behaviors observed in real situations. Also, little is known about

the relationships between engaged behaviors and the motivational impact of gamification.

To answer these questions, we conducted a large-scale field study in ecological conditions, involving 257 students (aged between 13-14 years' old) in 12 classes, from 4 different middle schools. The students completed 10 math lessons of approximately 40 minutes on a gamified learning environment developed for a research project named LudiMoodle. They were informed before the course that there was no formal assessment either during or at the end of the course, thus enabling us to identify the impact of the gamified 
learning environment without external influences such as final grades. This approach differs from many other studies in that we are able to identify behaviors that are not biased by motivations external to the gamified activity.

We performed statistical analyses on the interaction traces collected during the experiment to identify and validate a model of engaged behaviors when using a gamified digital learning environment. Our results show that we can distinguish two types of engaged behavior, which have different impacts on learners' motivation: (1) an achievement-oriented engagement that decreases learners' intrinsic motivation, (2) a perfection-oriented engagement that leads to an increase in learners' extrinsic motivation and a decrease in amotivation. These two types of behavior depend on whether learners are initially intrinsically motivated by the learning activity (or not) and on their level in the "achiever" dimension of their player profile. However, a third type of observed behavior, related to the time spent answering quizzes, cannot be characterized in terms of engagement. We believe that these findings contribute to a better understanding of how gamification affects learner behaviors during a gamified learning activity according to their profile, and how the different types of engaged behavior impact a gain or a loss of motivation for learners. Finally, we provide some recommendations for the design of gamified learning environments in terms of motivational affordances.

\section{Related work}

\subsection{Impact of gamified learning environments on learners}

In recent years, several studies have been conducted to evaluate the impact of game elements on learners' motivation in educational contexts. For example, Hanus and Fox [15] studied the impact of a 16-week gamified course on learners' intrinsic motivation, compared to a non-gamified course. Results revealed that students in the gamified course showed less intrinsic motivation, satisfaction, and autonomy over time than those in the non-gamified class. Similarly, Kyewski and Krämer [16] showed that learners' intrinsic motivation decreased over time when using a gamified online learning environment over a 5 -week period (the learning environment was gamified using a badge system).

However, other studies provide more mixed and even positive results. For example, Sailer et al. [17] showed that badges, leaderboards, and performance graphs had a positive effect on the satisfaction of competence needs, as well as on the perception of the meaning of tasks, while avatars, stories, 
and teammates had an effect on social relationships. Landers et al. [18] also studied the effect of using leaderboards for a gamified brainstorming task. Participants were randomly assigned to one of four different levels of goal setting (do your best, easy, difficult, and impossible), where a leaderboard represented their ranking. The presence of a leaderboard helped motivate participants to achieve the highest levels of performance, even without being forced to do so. Finally, Zainuddin [19] studied the difference between a gamified and a non-gamified flipped course. Performances were significantly better for the group with the gamified course, as was also the intrinsic motivation perceived by learners, especially with regard to feelings of competence and autonomy.

Van Roy et al. [20] analyzed the motivational processes underlying the motivational impact of gamification. This study is one of the first to distinguish the impact on different types of motivation, differentiating between intrinsic motivation, extrinsic motivation, amotivation, autonomous motivation, and controlled motivation. Over a long experimental period, they showed that all types of motivation evolved over time: they first decreased before increasing at the end of the course (except for amotivation, which increased at the start then decreased at the end, and controlled motivation, which remained stable, without returning to its initial level). The results illustrate the importance of the individual nature of motivational processes, and the importance of longitudinal motivational measures (not just the final outcomes).

However, as yet few studies have been conducted on the motivational processes involved when learners use a gamified learning environment. These processes are primarily analyzed through measures of learners' engagement. For instance, Ding et al. [21] conducted a first study on the impact of using a gamified online discussion tool $g E c h o L u$ on cognitive, emotional, and behavioral engagement. The results did not show any significant change in the dimensions of engagement between the middle and the end of the study. In line with these results, Landers et al. [18] showed that the engagement of learners achieving learning goals was the same with and without the presence of a leaderboard. Ding et al. [22] conducted a further study on the influence of the gamification approach on users' engagement in online discussions with the same $g E c h o L u$ environment. Unlike the previous study, the results showed that the gamification approach had a positive effect on all dimensions of users' engagement. Finally, da Rocha Seixas et al. [23] conducted a study on the effectiveness of badges offered on two platforms (ClassDojo and 
ClassBadges). They were able to classify students into four groups, which showed different types of engagement, observed through behaviors related to autonomy, participation, and collaboration.

These studies show a wide disparity in the results obtained when observing the impact of a gamified learning environment on learners, whether on motivation as a final result or on engagement as a process. Furthermore, as yet few studies have been conducted to understand the different types of engaged behaviors that can be observed when using such environments. In this paper, we contribute to the field by proposing a model of learners' engagement when using a gamified learning environment, and by analyzing the relationship between learners' motivation and engagement. In the following section, we shed light on the theoretical foundations that ground our analysis.

\subsection{Theoretical background on learners' motivation and engagement}

Most existing conceptual studies on gamification rely on the self-determination theory (SDT), a theory of human motivation initiated by Deci and Ryan $[24,25]$. Both psychologists developed this theory in order to understand the development and well-being of human psychology. This theory postulates that individuals have three basic psychological needs: competence, autonomy, and relatedness, and that humans strive to satisfy these three needs in order to improve their well-being. If these three needs are fulfilled, then their intrinsic motivation will be enhanced. Ryan and Deci [26] proposed to represent motivation as a continuum, from controlled motivation to autonomous motivation, starting from extrinsic motivation driven by external rewards, to intrinsic motivation linked to a high degree of autonomy of learners in their learning. One issue of gamification is then to generate extrinsic motivation through rewards, while gradually leading learners to increase their intrinsic motivation in order to make them increasingly autonomous in their learning. This mechanism is often used under the concept of motivational affordances of the digital environment.

The concept of "motivational affordance" was introduced by Zhang [27], inspired by the notion of "affordance", i.e. the capacity of an object to suggest its own use through the perception of possible actions. Applied to motivation, the concept corresponds to the properties of an object that supports users' motivational needs, thus enhancing their engagement and user experience. The concept is extended specifically to gamification by Deterding 
[1], who builds on Zhang's work to introduce the concept of situated motivational affordance. According to this approach, the situation in which the user is placed plays an important role in motivational affordances: it offers its own motivational characteristics (situational affordances) in addition to influencing the use, meaning and, consequently, the motivational affordances of the artifact in question (artefactual affordances).

According to this approach, in the context of learning through a digital learning environment, we can distinguish two motivational objects: learners' motivation for the learning activity (corresponding to situational affordances according to Deterding [1]), and their motivation for game elements (referring to artefactual affordances). The learning activity includes, for instance, the discipline (e.g. mathematics or French), the type of educational task (e.g. quiz, reading, writing), the content (e.g. text, video, animations). In particular, a learner who feels competent in a discipline or learning activity will tend to be motivated to carry it out.

These motivational sources are conditions for learners to engage in the gamified learning activity. Indeed, when learners' basic needs are fulfilled (referring to the SDT), they will tend to engage in the learning activity [28]. Engagement can thus be defined as the psychological investment and behavioral involvement of learners in learning activities [29], resulting from motivational affordances. Furthermore, engagement is a dynamic process that fluctuates over time; a learner may be engaged, disengaged, and reengaged in a course [3]. This perspective leads us to question the impact of learners' engaged behaviors during learning activities. We can assume that their engagement in the learning task could impact their level of motivation, depending on whether they feel competent, and have sufficient autonomy, and social relationships.

To positively influence learner engagement in the learning activity, we need to identify the degree and type of learners' engagement when interacting with the gamified learning environment. Engagement is a multidimensional concept composed of three complementary dimensions: cognitive, motivational/affective, and behavioral [30,2]. Motivational/affective engagement includes the interest, emotions, and values perceived by learners during learning activities. Cognitive engagement is related to the deployment of learning strategies: cognitive, self-regulated or resource management-related [31]. Behavioral engagement refers to the observable actions of the learner in completing a learning task [2].

Learners' actions are commonly observed through their interaction traces 
with the learning environment. Fincham et al. [7] identified several indicators of learners' engagement such as the number of weeks the student logs in, the number of unique videos watched, and the number of unique problem submissions. Motz et al. [32] used a rather exhaustive number of features of learners' activities with a Learning Management System to analyze learners' engagement, including time on pages, average page views, and average page views per session. In line with these studies, we focus on observable actions through the interactions of learners with the learning environment.

In conclusion, learners' motivation and engagement are two complex and interrelated concepts: learners' motivations can influence their engagement, and conversely, engagement in the activity can influence learners' level of motivation. In our study, which relies on the model of learners' engagement, we propose to further the relationships between learners' engagement and motivation by analyzing learners' interaction with the learning environment.

\subsection{Influence of player profiles on the impact of gamified learning environ- ments}

In addition to the gamified activity context, Nicholson [33] insists on taking into account the specificities of users (differences in experience, desires or skills) when designing gamified systems. Learners can have different preferences concerning game mechanics, as well as different motivations for learning $[34,11,10]$. Most systems therefore use profiles to categorize and classify learners, based on information such as their player type [35, 36], personality traits [37], and, more rarely, learners' motivation [38]), or more context-dependent information, such as learning styles [39, 40].

Player types can be defined as reasons why people play and enjoy games as a basis for classification [12]. Player type is a term first coined by Bartle in the 1990s [41] to categorize groups of MUD (multi-user dungeon) players. Bartle proposed four player types representing the kind of play style sought from the MUD experience: Explorers, Achievers, Killers, and Socializers, with game mechanics and elements for each. While the Bartle player types have seen some use in gamification settings [42], there are many other player profile models that are more recent, and appropriate for use in gamification.

Monterrat et al. [11] used the BrainHex typology to show that learners using game elements counter-adapted to their player profile perceived their game elements as more fun and useful than learners who used adapted game

elements. In a more recent study, Lavoué et al. [13] showed that providing learners with game elements corresponding to their player profile reduced the 
amotivation of those most engaged. Dos Santos et al. [43] showed that for some of the Brainhex [36] player types, game elements increased or decreased flow depending on learners' preferences for game mechanisms [44].

Nowadays, the most commonly used player type typology is Hexad [35], created specifically for gamification and that has proved to be more effective for gamification than other player profiles [45]. Based on the Self Determination Theory [25], this profile distinguishes six different categories: Philanthropists, Socializers, Free Spirits, Achievers, Players, and Disruptors and has been used in several recent studies. For example, Mora et al. [46] sorted learners into one of four gamified situations based on their Hexad profile scores. They reported an increase in behavioral and emotional engagement from students who were provided with game elements suited to their player type. Oliveria et al. [47] also evaluated learners' flow experience, but found no differences between learners depending on the game elements they used.

To the best of our knowledge, to date only a few studies have been conducted on the impact of learners' motivation for learning tasks when using a gamified learning environment. Hanus and Fox [15] showed that the effect of gamification on students' final exam results was influenced by the students' intrinsic motivation level, where students in the gamified course showed less motivation and obtained lower final exam results than those in the non-gamified course. Recently, Hassan et al. [40] adapted their system to learners' motivation for the task, identified based on a questionnaire adapted from the Academic Motivational Scale proposed by Vallerand et al. [48]. They observed an increase in course completion and motivation for learners who used game elements corresponding to their motivations.

In line with this research, our study addresses the question of the influence of player types and initial motivation for the learning task on learners' engagement with a gamified learning environment. We analyze these influences on different types of engaged behavior observed through interaction traces with the learning environment.

\section{Research questions}

In this paper, we aim at understanding more thoroughly the processes involved in learners' behaviors when using a gamified learning environment. Based on the literature, we hypothesize that we can observe different types of engaged behavior and that the latter are linked to an evolution in learner motivation during use of the gamified system. Our study is not limited to merely 
identifying a plausible model of learners' engaged behaviors. Rather, we aim to situate the proposed model as a mediator between individual learner characteristics and the variation in learners' motivation. We hypothesize that learners' engaged behaviors are influenced by their initial motivation for the course and by their player profile. Thus, we address the following research questions:

RQ1. Can we identify different types of engaged behavior through learner interaction traces with the gamified learning environment? We explore the use of trace data to build a latent variable model structure consistent with the different types of learners' engaged behaviors.

RQ2. To what extent, and in what ways, do engaged behaviors influence the variation in learners' motivation from the beginning to the end of the course? We conduct a statistical analysis (called path analysis, explained in detail in the next section) to investigate both how our model's structure is predictive of motivational outcomes, and the interdependencies of the latent variables within the model itself.

RQ3. How does learners' initial motivation influence the different types of engaged behavior? We perform a path analysis to investigate to what extent, and in what ways, the behaviors identified in answer to RQ1 are influenced by the initial motivation of learners.

RQ4. How does learners' player profile influence the different types of engaged behavior? We perform a path analysis to investigate to what extent, and in what ways, the behaviors identified in answer to RQ1 are influenced by the player types of learners.

\section{Learning environment}

The participants used a gamified version of the Moodle Learning Management System called "LudiMoodle" (see Fig. 1), developed within the scope of the LudiMoodle project. This project brings together researchers in computer science and in educational sciences, pedagogical designers, four middle schools, and the company Edunao. It aims at evaluating the impact of a gamified learning environment on learners' motivation and engagement according to his/her profile. In total, the environment proposes six different game elements that were designed in collaboration with the five teachers involved in the project. The six game elements used are described in section 4.2: Avatar, Badges, Progress, Ranking, Score, Timer. 


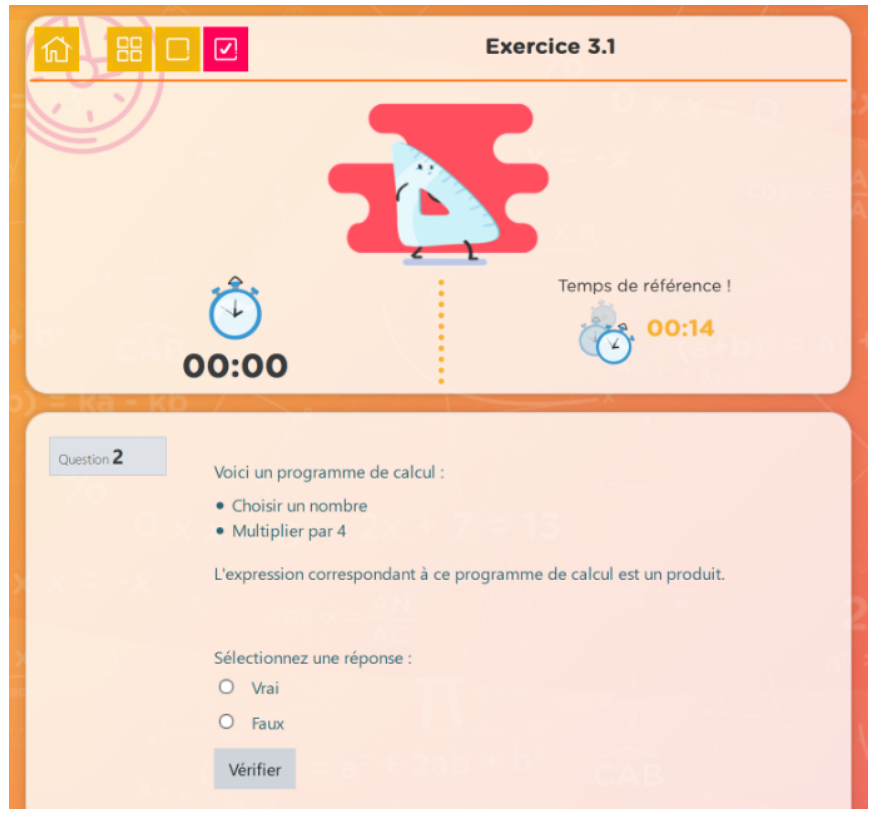

Figure 1: The Ludimoodle platform: example of a gamified quiz. The upper part shows a timer, while the lower part contains a quiz question.

\subsection{Learning content}

We built the learning content using a co-design method with the participating teachers in order to adhere as closely as possible to their usual teaching practices. In total, ten lessons were designed to cover the topic of basic algebra (calcul littéral in French). Each lesson is composed of 4 to 10 quizzes. The content proposed in the LudiMoodle system did not completely cover the lesson plan as it was only used for reinforcement exercises (as designed by the teachers during early co-design phases). Teachers had observed that, generally, these reinforcement exercises were not particularly appreciated by learners, as they found them to be boring or too repetitive. Teachers therefore wanted to make these types of exercise more engaging for learners.

Each lesson was conducted in the same way: 10-15 minutes of written notes (handed out by the teachers to ensure that learners had access to the same learning content), followed by 25-30 minutes for answering quizzes related to the lesson topic, using the LudiMoodle platform. To successfully complete a quiz and progress to the next one, learners had to answer at least $70 \%$ of all questions correctly. Learners used an individual tablet to access 
the quizzes. During this time, teachers answered questions asked by learners individually. In total, learners therefore used the LudiMoodle platform for 10 mathematics lessons.

\subsection{Game elements}

In total, six game elements were implemented in the gamified learning environment. Each of these game elements was designed either to entice learners to explore all quizzes (avatar and progress for example), answer quickly (notably for timer), and strive for a better performance - i.e. more correct answers to questions (badges, ranking, score for example).

\subsubsection{Avatar}

The avatar game element showed a goblin-like character that explored different universes (a different universe for each lesson). As the learner progressed in a lesson so they unlocked a different piece of clothing, or an item that the character was holding. There was one object to unlock per quiz (unlocked after the learner correctly answered at least $70 \%$ of the questions in the quiz).

\subsubsection{Badges}

The badge game element proposed three levels of badges per quiz. Once the learners had correctly resolved three different levels of questions in the quiz (generally 70-85-100\% of each quiz), they would unlock a new level of badge (bronze-silver-gold). An icon on the left-hand side showed how many badges the student unlocked for the current lesson.

\subsubsection{Progress}

This game element showed different colored spaceships traveling from the earth to the moon. Each lesson launched a new spaceship, and if the learner completed at least $70 \%$ of the lesson, the spaceship would land on the moon.

\subsubsection{Ranking}

Learners assigned to this game element could compare themselves to a fictional class of learners. The ranking game element showed a "race" where, as the learners answered questions correctly, they progressed in the race at the same pace as the other fictional learners. If they failed to answer a question correctly, they then fell back in the ranking. We calibrated the ranking system to ensure that a learner who completed at least $70 \%$ of a 
lesson would finish in the top $50 \%$ of the ranking to ensure they were not demotivated.

\subsubsection{Score}

Each correct answer given by the learners awarded them 1000 points. Each lesson had its own score counter, with a detailed view that showed how many points they had scored for each quiz. This allowed learners to pinpoint where they were missing points.

\subsubsection{Timer}

This game element showed a timer for each quiz. Learners were asked to try and beat a "reference time" for each question. Reference times were calculated based on the times for their previous questions in the same quiz. Each time a learner beat their reference time, an animation changed, with a character running faster and faster.

\section{Study design}

\subsection{Participants}

To answer our research questions, we ran a large-scale field study in real conditions as part of the LudiMoodle project. A total of 5 teachers (those involved in the design of the course) and 313 students (13-14 years' old) in 12 classes (an average of 25 students per class), from 4 different middle schools, participated in the study. We filtered students to keep data only for those who completed more than 10 quizzes. We also removed from our dataset students who did not correctly fill out all 3 questionnaires. As a result, we had a final total of 257 participants in our dataset (123 self-reported as female and 135 self-reported as male).

\subsection{Material and data}

\subsubsection{Engaged Behaviors}

Learners' interactions with the learning environment were tracked using the Moodle data logging system. All of the log traces were built using the following format: timestamp, learner ID, game element used by the learner, the name of the interaction, and optional attributes depending on the type of interaction (e.g. quiz number, question number, question result (fail or pass)). 
We then listed all possible learner actions with the learning environment to identify engaged behaviors. When defining our indicators, we were more interested in how learners interacted than how much they interacted. As we filtered learners based on the number of quizzes they completed, we could ensure they had a sufficient number of interactions with the learning content on which to base our analyses. For some of the indicators, we chose to use ratios (by dividing the count by the number of quizzes/questions attempted) instead of direct counts, since learners did not access the same number of quizzes. In fact, learners had to correctly answer $70 \%$ of the questions in a quiz to access the next quiz in a lesson. At the start of a new lesson, teachers unlocked the first quiz of the lesson for all learners, meaning that for some learners, the previous lesson's quizzes were not always fully completed (or attempted). We calculated the following indicators:

- AvgQuestionTime: average time taken to answer a question (for all question attempts). This was calculated for the first attempt at each question (as questions did not change on successive attempts).

- PassedFirstQuizRatio: ratio of quizzes successfully completed at the first attempt, including bonus quizzes (i.e. the number of successful quizzes divided by the number of quizzes attempted). A quiz was successfully completed if at least $70 \%$ of questions were correctly answered.

- PerfectFirstQuizRatio: ratio of quizzes perfectly completed at the first attempt, including bonus quizzes (i.e. the number of prefect quizzes at the first attempt divided by the number of quizzes attempted). A quiz was counted as "perfectly completed at the first attempt" if $100 \%$ of the questions were correctly answered at the first attempt.

- QuestionRatioInitiale: correct question ratio (i.e. the number of correct answers divided by the total number of questions in a quiz), averaged over the total number of quizzes attempted. This was calculated for the first attempt at each quiz.

- NBonusQuiz: number of distinct bonus quizzes completed (each different bonus quiz was only counted once).

- AverageRestartedTimes: average number of times a successfully completed quiz (> 70\%) was restarted (including quizzes that had been restarted at least once, and bonus quizzes). For example, learner L1 
completed the following quizzes: Q1:70\%, Q2:85\%, Q3:50\%, and then restarted Q1 2 times, Q2 3 times, and Q3 1 time. Learner L1 would have the following "AverageRestartedTimes": $(2+3) / / 2=2.5$ (two restarted quizzes that were restarted a total of five out of two completed quizzes).

- RestartedQuizzesRatio: ratio of successfully completed quizzes that were restarted, and bonus quizzes. Going back to the previous example: Q1:70\%, Q2:85\%, Q3:50\%, Learner L1 restarted Q1 2 times, Q2 3 times, and Q3 1 time. Learner L1 would have the following "RestartedQuizzesRatio": $2 / 2=1$ (two restarted quizzes out of two completed quizzes).

- StreakRatio: average number of quizzes attempted in a session without restarting a successfully completed quiz divided by the number of quizzes attempted in the session.

\subsubsection{Profile questionnaires}

We identified participants' motivation and player profile using questionnaires. Regarding learners' motivation, we used the Academic Motivation Scale (AMS) proposed by Vallerand et al. [48] (inspired by SDT [25]). The AMS evaluates seven dimensions of motivation: three for intrinsic motivation (IM), three for extrinsic motivation (EM), and one for amotivation (AM). Each of these dimensions identifies the reasons why someone would perform an activity (we provide an example of one of the questions asked for each dimension):

- Intrinsic Motivation for Knowledge (IMK), i.e. performing an activity for the pleasure and satisfaction of doing something new: "I like learning new things"

- Intrinsic Motivation for Accomplishment (IMA), i.e. performing an activity for the pleasure of overcoming a challenge: "I like to see that I am able to solve problems"

- Intrinsic Motivation for Stimulation (IMS), i.e. performing an activity for fun or excitement: "I really like math"

- Identified Regulation (ID), i.e. performing an activity in order to achieve precise objectives regarding their future: "I will be able to choose my future studies thanks to math" 
- Introjected Regulation (IR), i.e. performing an activity to avoid shame or increase self-esteem: "I want to prove that I can do well in math"

- External Regulation (ER), i.e. performing an activity to gain some kind of external rewards: "I want to get a good grade"

- Amotivation(AM), i.e. the absence of intention to perform an activity: "I don't know why I go to math class, I feel like I'm wasting my time"

For the analyses, we grouped the intrinsic motivations (IM), just as in other studies [49], so as to dissociate only the self-determined types of motivation (IM and EM by identified regulation) and the non-self-determined types of motivation (EM by introjected and external regulations, and amotivation).

We identified learners' player profiles using a translated version of the original Hexad questionnaire [35], which defines six dimensions:

- Socializers, motivated by Relatedness: "Interacting with others is important to me". They want to interact with others and create social connections.

- Free Spirits, motivated by Autonomy and Self-expression: "It is important to me to follow my own path". They want to create and explore.

- Achievers, motivated by Mastery: "I like overcoming obstacles". They are looking to learn new things and improve themselves. They want challenges to overcome.

- Philanthropists are motivated by Purpose and Meaning: "It makes me happy if I am able to help others". This group is altruistic, wanting to give to other people and enrich the lives of others in some way with no expectation of reward.

- Disruptors are motivated by Change: "I like to provoke". In general, they want to disrupt the system, either directly or through other users, to force positive or negative change.

- Players are motivated by Rewards: "I like competitions where a prize can be won". They will do what is needed of them to collect rewards from a system. They are in it for themselves. 
Instead of determining the dominant player type for each learner, we saved the score of each Hexad dimension (Achievers, Players, Socializers, Free Spirits, Disruptors, and Philanthropists) in our dataset.

\subsubsection{Procedure}

Participants participated in 10 lessons of approximately 40 minutes on the LudiMoodle learning environment. Each lesson was conducted in the same way: 10-15 minutes of written notes (the written notes were handed out to learners by the teachers to ensure that they had access to the same content), followed by 25-30 minutes for answering quizzes related to the lesson topic. Learners used an individual tablet to access the quizzes. Teachers answered technical questions asked by learners individually.

Learners had to answer both the AMS and Hexad questionnaires before the first session (pre-test). After the last session (post-test), they only had to answer the AMS questionnaire. We were able to measure their initial motivation, final motivation, and the variation in motivation for each type of motivation.

We should mention that learners were intentionally not evaluated either during the experiment or at the end. We wanted to be able to measure learners' motivation without any external influence on motivation as a result of a final grade.

\subsubsection{Statistical method}

The data from all ten sessions were aggregated and randomly split into two equal sub-samples. For the AvgQuestionTime indicator, we converted the "hh:mm:ss" dates into the number of seconds and reduced the total variance of some of the larger values (division of AvgQuestionTime by 1000) to facilitate our analysis.

To answer our first research question and identify a latent variable model structure, an exploratory factor analysis (EFA) [50] was used on the first half of the dataset. While the factor structure was identified via an iterative process, whereby variables that did not load or exhibited factor loadings greater than 1 were excluded [51], the number of factors was selected via a parallel analysis scree plot. In brief, a parallel analysis involves generation of a random dataset of the same dimensions as the data being analyzed. Factor analysis is then performed on the random data to extract eigenvalues. To avoid bias, this process is repeated 20 times and, for each eigenvalue, an average is taken. These random eigenvalues are then compared with the 
eigenvalues of the real data, and factors in the real data are only retained if their eigenvalues are greater than the eigenvalues from the random data [52]. This analysis was conducted using the Psych package in R. To allow for correlations between factors, oblimin rotation was used and, given the relative normality of our data, standardized coefficients were estimated using maximum likelihood [51]. This permitted the computation of a wide range of goodness of fit indices, and allowed testing for the significance of factor loadings and correlations, as well as the computation of confidence intervals [53]. Using this identified structure, confirmatory factor analysis (CFA) was then conducted on the second half of the data, using the Lavaan package in R. Since the data were treated as continuous, the MLR estimator (maximum likelihood estimation with robust standard errors) was used.

To answer the 3 other research questions, a partial least squares path modeling (PLS-PM) was fitted to the entire dataset using the model structure identified in the first analysis (in answer to RQ1). In addition to the factor structure, PLS specifies a path analysis enabling us to evaluate associations between variables. We were particularly concerned with the associations between our latent variables and 1) the variation in motivation, 2) the player profile, 3) the initial motivation. The unidimensionality of each factor is guaranteed by the loadings of each indicator, and the Dillon Goldstein's rho index: a value of 0.70 is often cited as an acceptable cutoff [54].

\section{Results}

\subsection{Model of engaged behaviors (RQ1)}

We conducted a series of analyses to address our first research question and investigate whether a latent variable model, consisting of our selected engagement indicators (see Section 5.2), could be identified. First, we conducted a parallel analysis on the first half of the dataset. The results suggested a three-factor structure as shown in Figure 2. According to the scree plots, the "elbow" of the graph where the eigenvalues seem to level off, the random dataset is located, and factors or components to the left of this point should be retained as significant. We then conducted an EFA on the same data using an iterative process, whereby variables that did not load or exhibited factor loadings greater than 1 were removed [51] (this was the case for NBonusQuiz and AverageRestartedTimes). A value of 0.65 is used as a cutoff for factor loadings, resulting in a model with 6 variables and 3 factors $(\mathrm{TLI}=1.029, \mathrm{RMSEA}=0.000$ (90\% CI: 0.000-0.022), $\mathrm{SRMR}=0.01)$. 
Standardized loadings for this model are reported in Table 1. The fit of the identified factor structure was then assessed on the second half of the dataset using CFA. This also resulted in a good fit to the dataset $\left(\chi^{2}(4, \mathrm{~N}\right.$ $=144)=5.266$, p-value $=0.261, \mathrm{CFI}=0.996, \mathrm{TLI}=0.986, \mathrm{RMSEA}=$ 0.060 (90\% CI: 0.000-0.182), SRMR =0.017). The estimated standardized solution (standardized loadings), and p-values (for testing the null hypothesis that the loading equals zero) may be found in Table 2. All non-standardized loadings were statistically significant $(p<0.05)$, and the absolute value of all standardized loadings is more than 0.5.

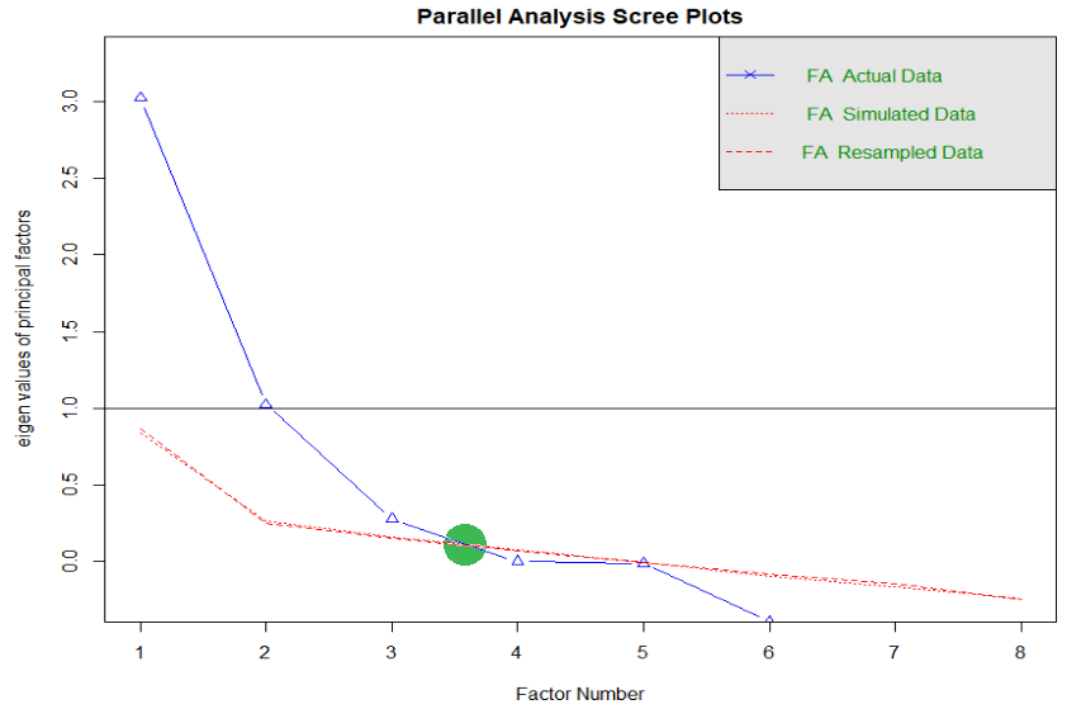

Figure 2: Parallel analysis scree plots of exploratory factor analyses, where the blue line shows the scree plot of the first half of our dataset, and the red line shows the scree plot of random data of the same size. The "elbow" of the graph is highlighted in green.

- The first factor groups three indicators: QuestionRatioInitiale, PassedFirstQuizRatio, and PerfectFirstQuizRatio. This corresponds to how successful learners were at answering quizzes at the first attempt.

- The second factor is composed of two indicators: RestartedQuizzesRatio and StreakRatio. This corresponds to learners who often redo successful quizzes, instead of doing many of them in a row.

- The third factor is composed of only one indicator (AvgQuestionTime) that corresponds to learners who answer quickly the questions of each 
Table 1: EFA Standardized Loadings

\begin{tabular}{lccc}
\hline Indicator & Factor1 & Factor2 & Factor3 \\
\hline AvgQuestionTime & & & -0.703 \\
QuestionRatioInitiale & 0.991 & & \\
PassedFirstQuizRatio & 0.964 & & \\
PerfectFirstQuizRatio & 0.865 & & \\
StreakRatio & & -0.653 & \\
RestartedQuizzesRatio & & 0.776 & \\
\hline
\end{tabular}

Table 2: CFA Standardized Loadings

\begin{tabular}{rlcccc}
\hline Factor & Indicator & Loadings & Std.Err & $R^{2}$ & $\mathrm{p}$ \\
\hline \multirow{2}{*}{ F1 } & QuestionRatioInitiale & 0.974 & 0.171 & 0.949 & 0.000 \\
& PassedFirstQuizRatio & 0.972 & 0.205 & 0.945 & 0.000 \\
& PerfectFirstQuizRatio & 0.886 & 0.187 & 0.785 & 0.000 \\
\hline \multirow{2}{*}{ F2 } & RestartedQuizzesRatio & 0.716 & 0.215 & 0.513 & 0.000 \\
& StreakRatio & -0.516 & 0.112 & 0.266 & 0.001 \\
\hline \multirow{2}{*}{ F3 } & AvgQuestionTime & -1.000 & 0.150 & 1.000 & 0.000 \\
\hline
\end{tabular}

quiz. This is associated neither with performances on quizzes nor with the number of quizzes completed or restarted.

To summarize, with regard to RQ1: "Can we identify different types of engaged behaviors through learners' interaction traces with the gamified learning environment?", we were able to show that the analysis of our interaction traces reveals a model that describes three types of learner behavior. The following sections investigate the relationships between these behavior types and motivational variation, initial motivation, and player type.

6.2. Relationship between engaged behaviors and variation in motivation (RQ2)

To address our second research question, we performed a path analysis (PLS-PM) to investigate the influence of each type of engaged behavior (represented through the latent variable model identified for RQ1) on the variation in each type of learner motivation. Table 3

shows the PLS path coefficients that reflect the influence that each factor of the identified model has on the variation in motivation score for each 
type of motivation. Regarding the unidimensionality of the factor structure, loadings are above the acceptable cutoff of 0.7 for all the variables in the model. The Dillon-Goldstein's rho index is 0.975 for factor $1,0.827$ for factor 2 , and 1 for factor 3. The structure and path coefficients of our PLS are shown in Figure 3. All the indices and loadings are above the acceptable cutoff of 0.7 .

Table 3: PLS path coefficients for each engagement factor of evolution of each type of motivation. Values in gray are not significant $(p>.05)$, values highlighted in dark gray are significant $(p<.05)$, and values highlighted in black are highly significant $(p<.001)$.

\begin{tabular}{lccc}
\hline & Factor 1 & Factor 2 & Factor 3 \\
\hline IMVar & -0.133 & 0.014 & 0.017 \\
AMVar & -0.042 & -0.319 & 0.072 \\
IDVar & -0.015 & 0.158 & -0.036 \\
IRVar & 0.052 & 0.070 & 0.009 \\
ERVar & 0.038 & 0.026 & -0.067 \\
\hline
\end{tabular}

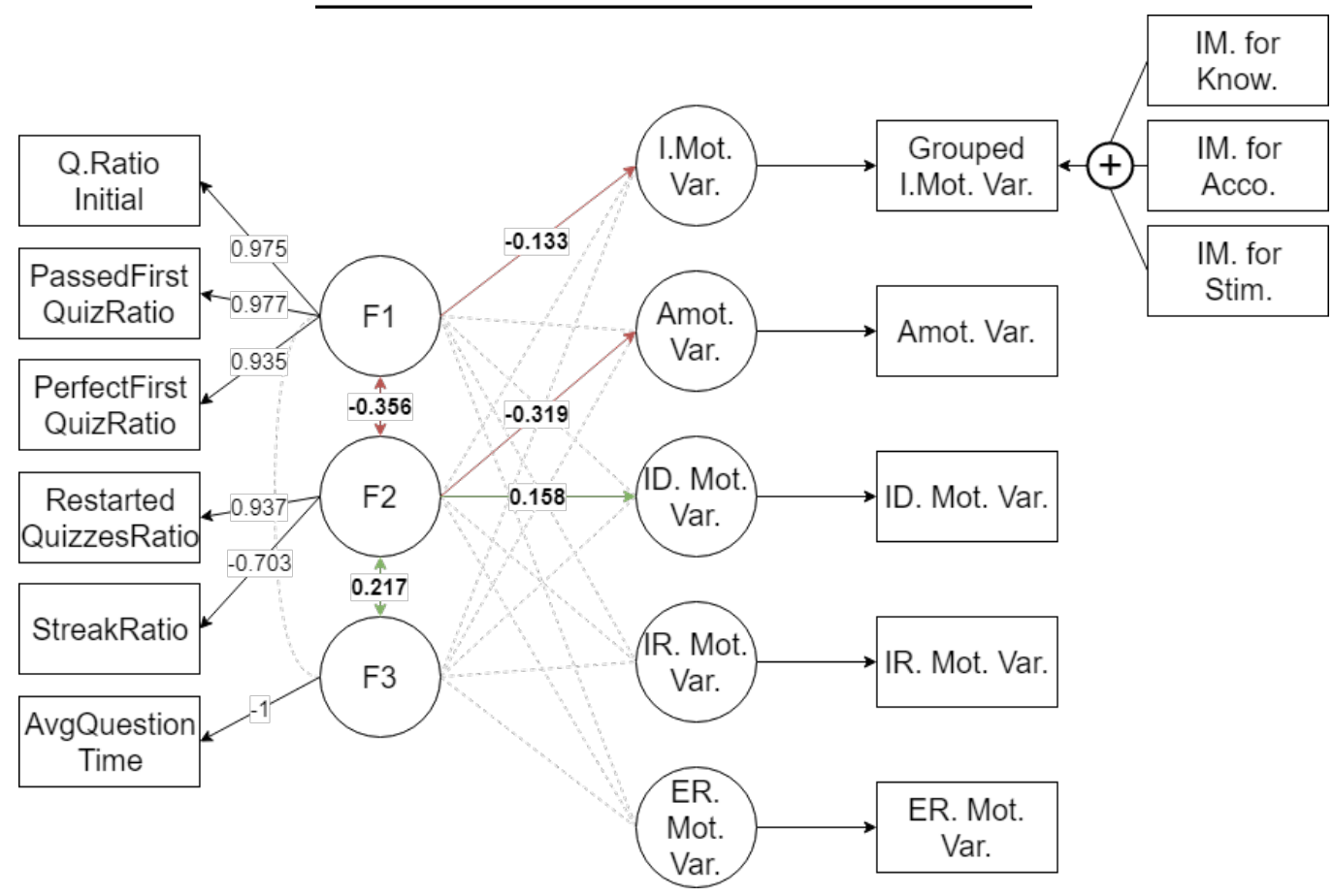

Figure 3: PLS of engagement factors with evolution of motivation. Single-headed arrows represent direct influences, while double-headed arrows represent correlations. 
The path analysis indicates that Factors 1 and 2 significantly influenced the variation in different types of motivation, while Factor 3 had no influence. Factor 1 negatively influenced the variation in intrinsic motivation, meaning that it led to a decrease in learners' intrinsic motivation. Factor 2 negatively influenced the variation in amotivation and positively influenced the variation in identified regulation. This meant that learners who repeated quizzes became more motivated to continue the activity in order to enhance opportunities for their future studies and career.

As shown in Figure 3, factors F1 and F2 are negatively correlated, while F2 and F3 are positively correlated.

To summarize, with regard to RQ2: "To what extent, and in what ways, do engaged behaviors influence the variation in learners' motivation from the beginning to the end of the course?", we found that one behavior (corresponding to F1) negatively influenced the variation in intrinsic motivation, and that another behavior (corresponding to F2) influenced both the variation in amotivation and the variation in identified regulation.

\subsection{Influence of initial motivation on engaged behaviors (RQ3)}

To answer our third research question, we conducted a second PLS-PM to investigate the influence of learners' initial motivation on the latent variable model we identified in Q1. Table 4 shows the PLS path coefficients that reflect the influence that players' initial motivation has on each factor of the identified model. Regarding the unidimensionality of the factor structure, loadings are above the acceptable cutoff of 0.7 for all the variables in the model. The Dillon-Goldstein's rho index is 0.975 for F1, 0.827 for F2, and 1 for F3. The structure and path coefficients of our PLS are shown in Figure 4. All the indices and loadings are above the acceptable cutoff of 0.7 . 
Table 4: PLS path coefficients for initial motivation of each engagement factor. Values in gray are not significant $(p>.05)$, values highlighted in dark gray are significant $(p<.05)$, and values highlighted in black are highly significant $(p<.001)$.

\begin{tabular}{lccccc}
\hline & IM & AM & ID & IR & ER \\
\hline Factor1 & 0.347 & 0.050 & 0.110 & -0.116 & -0.105 \\
Factor2 & -0.137 & 0.053 & -0.137 & 0.061 & -0.077 \\
Factor3 & 0.068 & -0.148 & 0.061 & -0.051 & -0.131 \\
\hline
\end{tabular}

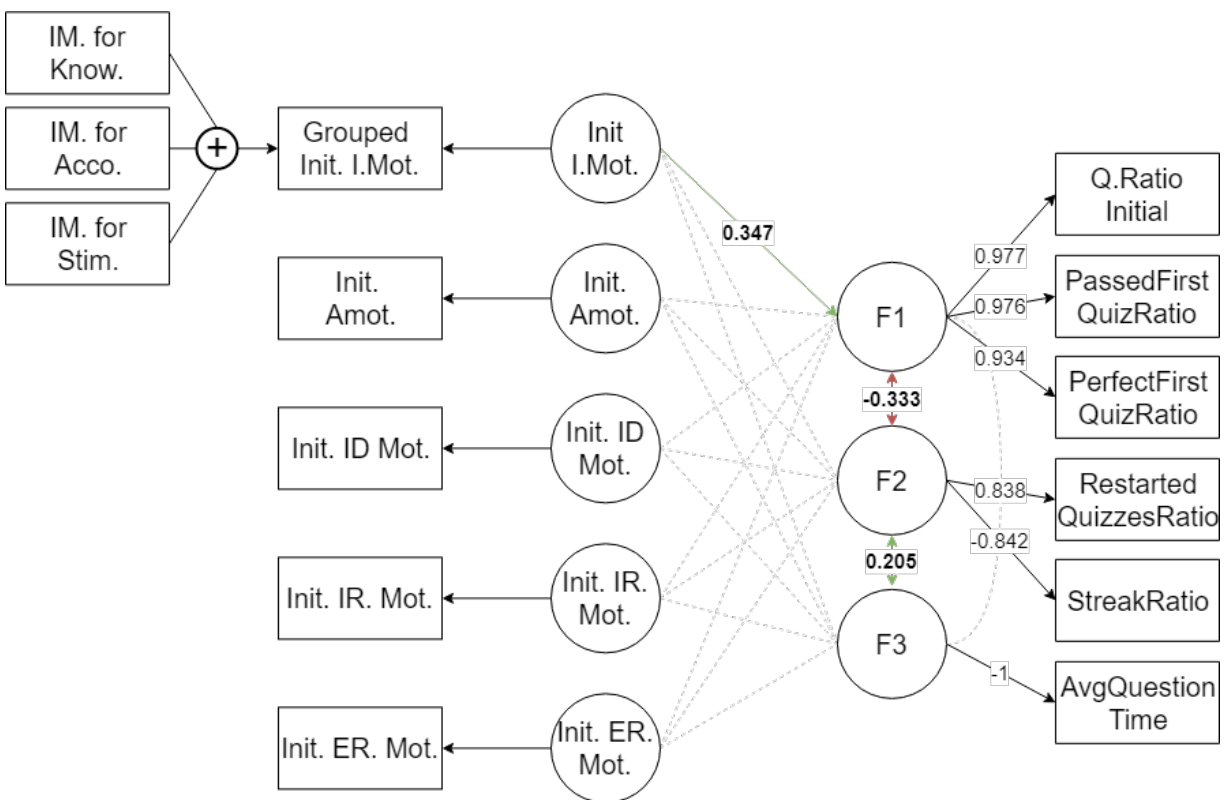

Figure 4: PLS of initial motivation with engagement factors. Single-headed arrows represent direct influences, while double-headed arrows represent correlations.

This second path analysis indicates that initial intrinsic motivation highly significantly positively influenced Factor 1 . This means that the more learners were intrinsically motivated at the beginning, the more they successfully answered quizzes at the first attempt. We observe no influence of the other types of initial motivation on the three factors.

Therefore, with regard to RQ3: "How does learners' initial motivation influence the different types of engaged behavior?", one type of motivation (intrinsic motivation) affects one type of engaged behavior (corresponding to 
F1).

\subsection{Influence of player profile on engaged behaviors (RQ4)}

To answer our fourth research question, we conducted a third path analysis to investigate the influence of learners' player profile on each type of engaged behavior. Table 5 shows the PLS path coefficients that reflect the influence that each dimension of the player profile has on each factor of the identified model. Regarding the unidimensionality of the factor structure, loadings are above the acceptable cutoff of 0.7 for all the variables in the model. The Dillon-Goldstein's rho index is 0.975 for factor $1,0.827$ for factor 2 , and 1 for factor 3 . The structure and path coefficients of our PLS are shown in Figure 5. All the indices and loadings are above the acceptable cutoff of 0.7 .

The achiever dimension positively influenced factor 1 , while it negatively influenced factor 2. This means that achievers aim at obtaining high performances at quizzes at the first attempt and at not redoing the quizzes they already attempted. The socializer dimension negatively influenced both factors 2 and 3, meaning that they do not redo quizzes and take more time to answer questions. Finally, the philanthropist dimension positively influenced factor 3, meaning that philanthropists tend to answer questions quickly.

To summarize, with regard to RQ4: "How does learners' player profile influence the different types of engaged behavior?", the path analysis indicates significant influences of the achiever, socializer, and philanthropist dimensions on all three factors F1, F2, and F3. 
Table 5: PLS path coefficients for player profile dimensions of each engagement factor. Values in gray are not significant $(p>.05)$, values highlighted in dark gray are significant $(p<.05)$, and values highlighted in black are highly significant $(p<.001)$.

\begin{tabular}{lcccccc}
\hline & Achi. & Player & Sociali. & Fr.Sp. & Disr. & Phil. \\
\hline Factor1 & 0.293 & -0.034 & -0.228 & 0.015 & 0.024 & 0.098 \\
Factor2 & -0.243 & -0.087 & 0.091 & 0.034 & 0.106 & 0.037 \\
Factor 3 & 0.051 & -0.046 & -0.198 & -0.003 & 0.101 & 0.189 \\
\hline
\end{tabular}

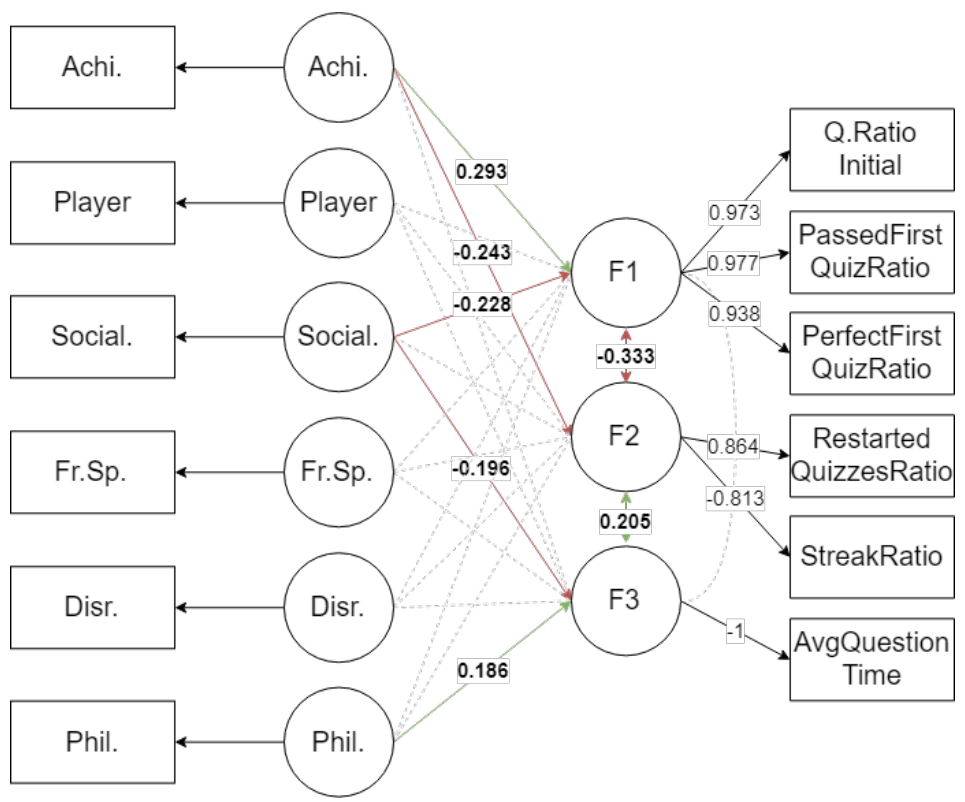

Figure 5: PLS of player profile with engagement factors. Single-headed arrows represent direct influences, while double-headed arrows represent correlations.

\section{Discussion}

\subsection{Different types of behavior in a gamified learning environment}

The three latent factors reveal three different types of behavior, which can be interpreted in light of their relationships with learners' motivation and player profile. In fact, analyzing our factor structure with three path analyses allowed us to investigate the extent to which our latent variables (representing engagement) were associated with a variation in learner motivation (represented by the difference between initial and final scores for 
each type of motivation), as well as their player profile. The following sections describe and name each of the behavior types. The first two are clearly related to different forms of engagement (Achievement-oriented or Perfectionoriented), whilst the third is only related to learners' speed (which, on its own, is not necessarily linked to a form of engagement). Finally, it is important to note that learners may exhibit one, multiple, or none of these different behavior types, meaning that it is possible that non-engaged learners did not follow any of these behavior types.

\subsubsection{Achievement-oriented engagement}

The first engagement factor corresponds to an achievement-oriented engagement. Learners who exhibit this type of behavior want to complete all quizzes with the best possible performance at the first attempt. They will generally complete a quiz (at least to $70 \%$ ) and progress to the next one. This is backed by the results in section 6.4 with regard to the influence of the achiever dimension, and in section 6.3 with regard to the influence of learners' initial intrinsic motivation. Both of these profile dimensions have a significantly positive influence on this kind of engaged behavior. It can therefore be expected that learners, who are more intrinsically motivated by the learning content, will look for new content to learn, always trying new quizzes. Also, as per the definition of the Achiever dimension: "They are looking to gain knowledge, learn new skills, and improve themselves. They want challenges to overcome." [35], this fully supports the engaged behavior observed here through Factor 1. As they are typically motivated by mastery, we can expect achievers to want to complete every challenge of our learning environment and to be the best at each one.

Looking at the other dimensions of the learner profile, we noticed in section 6.4 a negative influence from the Socializer dimension. We believe that this is due to a lack of "socially-oriented game elements" on our learning platform. This means that learners with high socializer scores would be unable to find game elements suited to their preferences and therefore would not be motivated to progress through the learning content. This choice was made in accordance with teachers, who thought that social game elements would disturb the learning activity in the classroom.

As presented in section 6.2, we observed a decrease in intrinsic motivation, linked to this type of engagement. We believe this may be due to two reasons. For highly intrinsically motivated learners, it is acknowledged that gamification decreases their intrinsic motivation. In fact, the theoretical 
literature highlights the negative effects of rewards on intrinsic motivation $[55,56]$. This is also observed in several studies that show a negative impact of gamification on initially intrinsically motivated learners [15, 16]. High achievers may have perceived a lack of difficult learning challenges presented by our application. When they failed to encounter any particular difficulties after completing the required quizzes, they might have lost some intrinsic motivation for the learning task.

\subsubsection{Perfection-oriented engagement}

The second factor reveals a perfection-oriented engagement. This corresponds to learners who want to improve their performances on quizzes and who aim at completing each quiz to $100 \%$ by redoing them. As we did not observe any links between initial motivation (section 6.3), we believe this engagement to be typical of learners who are neither particularly motivated by the learning task nor demotivated by it. We could have thought that high "achievers" also behave in relation to this second factor, given that the definition states that they want to improve themselves [35]. However, section 6.4 showed a negative influence of the achiever dimension on this type of behavior. Achievers would thus be more motivated by discovering new content and performing well in new challenges than by improving their performance on the same quizzes.

When looking at the effect that this type of engaged behavior had on learners' motivation, section 6.2 shows that learners who behaved accordingly not only lost amotivation, but also gained in identified regulation, a type of motivation defined as being self-determined (like intrinsic motivation). This finding is important as the gamified learning environment would lead learners, not initially motivated by the learning activity, to become more motivated to continue the learning activity. This would then be coupled with the wish to improve themselves to provide more opportunities for their future studies and career. Referring to the literature, badges, ranking, and scores may have increased the feeling of learners' competence, as in the studies reported in $[17,19]$, and incited them to attain the highest levels of performance [18].

This positive impact would be applicable only for learners who are not motivated by achievement (high achiever learners), i.e. discovering and completing the most quizzes. In fact, the negative correlation observed in section 6.2 between factors 1 and 2 means that, the more learners adopt an achievement-oriented engagement, which tends to discover the most quizzes with a high performance at the first attempt, the less they behave accord- 
ing to a perfection-oriented engagement to increase their performance on the same quizzes. This result reinforces the relevance of distinguishing these two different types of engagement.

\subsubsection{Behaviors linked to answering speed}

The third factor represents how quickly learners answer the questions in quizzes. A first finding of note, presented in section 6.3 , is that none of the initial motivations had any influence on this factor, meaning that the speed of answering questions does not correspond to a high or low initial motivation of learners. Regarding player types (section 6.4), the higher a learner's philanthropist score, the faster they answered questions, while the higher a learner's socializer score, the slower they answered questions. For high socializer learners, this may be due to a lack of social interaction game elements on the platform, just as observed for achievement-oriented behaviors. As a reminder, philanthropists are "altruistic and willing to give and help others without expecting a reward" [35]. Teachers commented on a certain category of learners who quickly finished answering questions, only to go and help their classmates, by correcting errors or giving tips. We could assume that the philanthropists exhibited this type of behavior.

When we look at the variations in motivation observed with this third factor (section 6.2), we can see that this behavior had no influence, implying that it cannot be used either as predictive of learners' variation in motivation or as being influenced by learners' initial motivation. Consequently, we are not able to classify this indicator as a type of engagement in our context. This is contradictory to other studies in the learning analytics domain that use this indicator as a predictor of learners' engagement. Further studies would be necessary to identify the specific impact of gamification on this particular behavior.

It is noteworthy that this factor is slightly associated with factor 2 (perfectionoriented engagement). This means that there is a subset of learners who quickly answer questions at the first attempt of a quiz, then sometimes go back and correct their mistakes with the desire to increase their performance

on this quiz. We could think that these learners are interested in the feeling of "freedom to fail", sometimes reported as a common principle of gamification $[57,58]$. "Freedom to fail" means giving learners the opportunity to experiment with the environment without fearing failure. For example, learners were free to answer questions incorrectly, then reflect on their answers, figure out where they went wrong, and restart the quiz. The correlation between 
this factor and factor 1 is not significant, meaning that answering questions quickly has no influence on achievement-oriented behavior, and vice versa.

\subsection{Implications for the design of gamified learning environments}

Our results provide useful insights into how motivation and learners' characteristics are linked to engaged behaviors, and how to take this into account when designing a gamified learning environment in the educational context. In this section, we discuss the design of motivational affordances, including both aspects: situational affordances (the learning activity), and artefactual affordances (the game elements). We then discuss another complementary approach, adaptive gamification, which aims at adapting the game elements to the learners' profile.

\subsubsection{Design of motivational affordances}

Our analysis showed that two different types of engagement, achievementoriented and perfection-oriented behaviors, emerged when interacting with the gamified learning environment. We showed that these behaviors are influenced by two aspects of learners' characteristics: their initial intrinsic motivation and their player profile, and more especially, the achiever dimension. Regarding motivational affordances, first we believe that the situational affordances offered by the learning environment engaged only learners with high intrinsic motivation, and that these learners adopted an achievement-

oriented behavior. Second, learners with high achiever levels tend to adopt achievement-oriented behaviors more than perfection-oriented behaviors. We believe that this was induced by the artefactual affordances of the system, with a game element design that impacted learners' behaviors. In the gamified environment, the rules for triggering game element updates were based on the completion of quizzes: completing a quiz above $70 \%$ allowed learners to access new quizzes and to discover new aspects of game elements (for instance, a new category of badge or a new universe for the avatar). We believe that this mechanism reinforced achievers' inclination to discover new content rather than to improve previous performances, thus favoring achievementoriented behaviors.

As highlighted in section 2.2, one of the challenges of gamification consists in generating extrinsic motivation through rewards, while gradually leading learners to increase their intrinsic motivation in order to make them more autonomous in their learning. We observed that perfection-oriented behaviors led to an increase in extrinsic motivation (increase in identified motivation 
and decrease in amotivation). Although it failed to increase learners' intrinsic motivation, the increase in identified motivation (which is considered to be a self-determined type of motivation) is an encouraging result. We could hypothesize that, in the long term, this could turn into intrinsic motivation. On the other hand, achievement-oriented engagement led to a decrease in intrinsic motivation. We believe that the learning environment did not offer enough quizzes and that it provided insufficiently rich content or questions that were too easy to solve, thus failing to increase (or to sustain, depending on the initial level) intrinsic motivation.

These findings argue that both situational and artefactual affordances should be designed with care, take into account the different characteristics of learners, and promote engaged behaviors enhancing all types of motivation (above all, by avoiding decrease in intrinsic motivation). In this case, situational affordances should sustain intrinsic motivation (especially for knowledge and accomplishment), providing appropriately difficult challenges for those learners that want and seek them. This can be applied for both intrinsically motivated and high achiever learners, who share a love of challenges. Artefactual affordances should be designed to promote specific engaged behaviors. In our context, perfection-oriented behaviors led to a gain in extrinsic motivation. However, achievement-oriented behaviors could also be supported if the situational affordances provide sufficient challenges and diversity of contents. Finally, the design of meaningful game elements should provide both artefactual and situational affordances, making sense to learners in the context of their learning activity and thus increasing their feeling of competence and autonomy.

\subsubsection{Adaptive gamification}

The methods and tools used to design game elements are thus useful for taking into account learners' characteristics and the learning situation before their integration into the digital learning environment. As a complementary approach, adaptive gamification can be used to adapt game elements to learners' profiles.

The field of adaptive gamification in education is still in its infancy [12]. To date, it is limited to an initial adaptation, consisting of allocation of game elements before utilization of the digital environment. While learners' motivation for the learning activity is key to the design of motivational affordances, as yet only two studies have looked into the impact of taking into account this characteristic during the adaptation process. Roosta et al. 
[38] showed positive results of this adaptation on learners' participation and performance. Hallifax et al. [14] showed that tailoring to both player type and motivation profiles can improve intrinsic motivation and decrease amotivation, compared to a single adaptation based only on learners' motivation. Moreover, learners' engagement fluctuates over time, and a game element that is engaging at the beginning of the course may no longer be effective after a few sessions. A dynamic adaptation of gamification would then be necessary, but the question arises of when to adapt and according to which criteria?

Gamification can have different purposes for learners, for instance to increase their level of performance or their engagement. Depending on the purpose, the data to collect will not be the same for triggering adaptation: for example, the results of a quiz to adapt to learners' performance or the number of interactions with the learning environment to adapt to their participation. Likewise, since there are several types of engaged behavior, a dynamic adaptation according to learners' engagement requires implementation of multi-modal sensors (e.g. interaction traces, emotion recognition, questionnaires)

\subsection{Limitations of the study}

We identified a few limitations of our study. First, our experiment was conducted at secondary school level, involving learners of the same age carrying out specific pedagogical activities (quiz), and was solely focused on mathematics. It is now well-known that the motivational impact of certain game elements varies according to the user activity or the domain of gamified systems [45]. Thus, other studies would be necessary to validate our model of engagement, i.e. the engaged behaviors identified in this context. However, the approach we propose for defining the different factors of engagement is context-independent and can be replicated.

Second, in our study we decided to exclude all grades and questionnaires on learners' knowledge to prevent this from influencing their motivation. We believe that this makes our findings easier to use by researchers or pedagogical engineers in their practices. Further studies would be necessary to investigate the relationships between engaged behaviors induced by the utilization of the gamified learning environment and a gain in learners' knowledge in the domain (Algebra).

Third, our study was conducted over ten learning sessions. We chose a relatively short period of time, thus enabling us to control the experimen- 
tal settings. With a study over a much longer period of time, we would surely observe different engaged behaviors. Indeed, variety is important in a learning situation, and other types of activities would be necessary to keep learners interested and engaged. However, they would provide different types of interaction and therefore different types of observable behavior. Furthermore, as learners' motivation varies according to the subject and the learning activities, the application of our approach would require the motivation questionnaire to be submitted before each course that introduces a new subject.

\section{Conclusion}

In this paper, we presented the results of a large-scale study on the impact of a gamified learning environment on learners' engaged behaviors and on the factors that influence these behaviors. Our study ran for close to six weeks in four different middle schools in France. We analyzed the data used by 257 learners from twelve different classes, from over 10 specifically designed mathematics lessons, gamified using six different game elements.

Our main contribution, in answer to RQ1, is a model of engagement that distinguishes two types of engaged behavior: an achievement-oriented engagement for initially intrinsically motivated learners or high achiever learners, and a perfection-oriented engagement for low achiever learners. A third type of behavior has been observed corresponding to learners who answer questions very quickly. We were not able to classify this indicator as a type of engagement. Moreover, this is contradictory to other studies in the learning analytics domain that use this indicator as a predictor of learners' engagement. Regarding RQ2, we highlight that each type of engaged behavior has a specific impact on a gain or loss of learners' motivation during the learning activity. With regard to RQ3, we show that intrinsic motivation positively influences achievement-oriented engagement. Finally, in answer to RQ4, we show that three dimensions of the player profile (achiever, socializer, and philanthropist) influence all behavior types.

This model contributes to a better understanding of 1) how gamification can affect learners' engaged behaviors during the learning activity according to their initial motivation and player profile, 2) how the different types of engagement impact learners' motivation. Although learners' engagement may differ according to the context, the proposed approach can be applied in any context to investigate learners' behaviors. These results open up new perspectives in terms of motivational affordance design and dynamic adaptation 
of gamification based on learners' interaction traces with the learning environment. Our future works will be directed toward the recommendations of dynamically adapted game elements in learning situations, for example expanding our work presented in Hallifax et al. [59].

\section{Acknowledgments}

This work is part of the LudiMoodle project financed by the e-FRAN Programme d'investissement d'avenir. We would also like to thank the teachers and students who participated in this study.

\section{References}

[1] S. Deterding, D. Dixon, R. Khaled, L. Nacke, From game design elements to gamefulness: defining gamification, in: Proceedings of the 15th international academic MindTrek conference: Envisioning future media environments, ACM, 2011, pp. 9-15.

[2] J. A. Fredricks, P. C. Blumenfeld, A. H. Paris, School Engagement: Potential of the Concept, State of the Evidence, Review of Educational Research 74 (1) (2004) 59-109.

[3] H. L. O'Brien, E. G. Toms, What is user engagement? A conceptual framework for defining user engagement with technology, Journal of the American Society for Information Science and Technology 59 (6) (2008) 938-955.

[4] P. Bouvier, E. Lavoué, K. Sehaba, Defining engagement and characterizing engaged-behaviors in digital gaming, Simulation \& Gaming 45 (4-5) (2014) 491-507.

[5] M. Cocea, S. Weibelzahl, Log file analysis for disengagement detection in e-Learning environments, User Modeling and User-Adapted Interaction 19 (4) (2009) 341-385.

[6] M. Filsecker, M. Kerres, Engagement as a volitional construct: A framework for evidence-based research on educational games, Simulation \& Gaming 45 (4-5) (2014) 450-470. 
[7] E. Fincham, A. Whitelock-Wainwright, V. Kovanović, S. Joksimović, J.-P. van Staalduinen, D. Gašević, Counting clicks is not enough: Validating a theorized model of engagement in learning analytics, in: Proceedings of the 9th International Conference on Learning Analytics \& Knowledge, 2019, pp. 501-510.

[8] K. Mongkhonvanit, K. Kanopka, D. Lang, Deep Knowledge Tracing and Engagement with MOOCs, in: Proceedings of the 9th International Conference on Learning Analytics \& Knowledge, LAK19, ACM, New York, NY, USA, 2019, pp. 340-342.

[9] R. Orji, L. E. Nacke, C. DiMarco, Towards personality-driven persuasive health games and gamified systems, in: Proceedings of SIGCHI Conference on Human Factors in Computing Systems, 2017.

[10] C. Lopez, C. Tucker, Towards personalized adaptive gamification: A machine learning model for predicting performance, IEEE Transactions on Games (2018).

[11] B. Monterrat, E. Lavoue, S. George, Adaptation of gaming features for motivating learners, Simulation \& Gaming 48 (5) (2017) 625-656.

[12] S. Hallifax, A. Serna, J.-C. Marty, e. Lavoue, Adaptive Gamification in Education: A Literature Review of Current Trends and Developments, in: Transforming Learning with Meaningful Technologies, Springer International Publishing, Cham, 2019, pp. 294-307.

[13] E. Lavoue, B. Monterrat, M. Desmarais, S. George, Adaptive Gamification for Learning Environments, IEEE Transactions on Learning Technologies 12 (1) (2019) 16-28.

[14] S. Hallifax, E. Lavoué, A. Serna, To tailor or not to tailor gamification? an analysis of the impact of tailored game elements on learners' behaviours and motivation, in: 21th International Conference on Artificial Intelligence in Education, 2020, pp. 216-227.

[15] M. D. Hanus, J. Fox, Assessing the effects of gamification in the classroom: A longitudinal study on intrinsic motivation, social comparison, satisfaction, effort, and academic performance, Computers \& Education 80 (2015) 152-161. 
[16] E. Kyewski, N. C. Krämer, To gamify or not to gamify? An experimental field study of the influence of badges on motivation, activity, and performance in an online learning course, Computers \& Education 118 (2018) 25-37.

[17] M. Sailer, J. U. Hense, S. K. Mayr, H. Mandl, How gamification motivates: An experimental study of the effects of specific game design elements on psychological need satisfaction, Computers in Human Behavior 69 (2017) 371-380.

[18] R. N. Landers, K. N. Bauer, R. C. Callan, Gamification of task performance with leaderboards: A goal setting experiment, Computers in Human Behavior 71 (2017) 508-515.

[19] Z. Zainuddin, Students' learning performance and perceived motivation in gamified flipped-class instruction, Computers \& Education 126 (2018) 75-88.

[20] R. van Roy, B. Zaman, Unravelling the ambivalent motivational power of gamification: A basic psychological needs perspective, International Journal of Human-Computer Studies (2018).

[21] L. Ding, C. Kim, M. Orey, Studies of student engagement in gamified online discussions, Computers \& Education 115 (2017) 126-142.

[22] L. Ding, E. Er, M. Orey, An exploratory study of student engagement in gamified online discussions, Computers \& Education 120 (2018) 213226.

[23] L. da Rocha Seixas, A. S. Gomes, I. J. de Melo Filho, Effectiveness of gamification in the engagement of students, Computers in Human Behavior 58 (2016) 48-63.

[24] E. L. Deci, R. M. Ryan, The general causality orientations scale: Selfdetermination in personality, Journal of research in personality 19 (2) (1985) 109-134.

[25] E. Deci, R. Ryan, The "what" and "why" of goal pursuits: Human needs and the self-determination of behavior, Psychological inquiry 11 (4) (2000) 227-268. 
[26] R. M. Ryan, E. L. Deci, Intrinsic and extrinsic motivations: Classic definitions and new directions, Contemporary educational psychology 25 (1) (2000) 54-67.

[27] P. Zhang, Technical opinion Motivational affordances: Reasons for ICT design and use, Communications of the ACM 51 (11) (2008) 145-147.

[28] P. Bouvier, K. Sehaba, Lavoué, A trace-based approach to identifying users' engagement and qualifying their engaged-behaviours in interactive systems: Application to a social game, User Modeling and User-Adapted Interaction 24 (5) (2014) 413-451.

[29] J. J. Appleton, S. L. Christenson, M. J. Furlong, Student engagement with school: Critical conceptual and methodological issues of the construct, Psychology in the Schools 45 (5) (2008) 369-386.

[30] E. A. Linnenbrink, P. R. Pintrich, The role of self-efficacy beliefs in student engagement and learning in the classroom, Reading \& Writing Quarterly: Overcoming Learning Difficulties 19 (2) (2003) 119-137.

[31] P. R. Pintrich, A Conceptual Framework for Assessing Motivation and Self-Regulated Learning in College Students, Educational Psychology Review 16 (4) (2004) 385-407.

[32] B. Motz, J. Quick, N. Schroeder, J. Zook, M. Gunkel, The Validity and Utility of Activity Logs As a Measure of Student Engagement, in: Proceedings of the 9th International Conference on Learning Analytics \& Knowledge, LAK19, ACM, 2019, pp. 300-309.

[33] S. Nicholson, A user-centered theoretical framework for meaningful gamification, Games+ Learning + Society 8 (1) (2012) 223-230.

[34] J. Hamari, J. Koivisto, H. Sarsa, Does Gamification Work? - A Literature Review of Empirical Studies on Gamification, in: 47th Hawaii international conference on system sciences, 2014, pp. 3025-3034.

[35] A. C. Marczewski, Even Ninja Monkeys like to play, CreateSpace Indep. Publish Platform, Charleston (2015).

[36] L. E. Nacke, C. Bateman, R. L. Mandryk, BrainHex: A neurobiological gamer typology survey, Entertainment Computing 5 (1) (2014) 55-62. 
[37] L. R. Goldberg, An alternative "description of personality": the big-five factor structure., Journal of personality and social psychology 59 (6) (1990) 1216.

[38] F. Roosta, F. Taghiyareh, M. Mosharraf, Personalization of gamification-elements in an e-learning environment based on learners' motivation, in: 8th International Symposium on Telecommunications (IST), 2016, pp. 637-642.

[39] M. Denden, A. Tlili, F. Essalmi, M. Jemni, Educational Gamification Based on Personality, in: 2017 IEEE/ACS 14th International Conference on Computer Systems and Applications (AICCSA), 2017, pp. 13991405 .

[40] M. A. Hassan, U. Habiba, F. Majeed, M. Shoaib, Adaptive gamification in e-learning based on students' learning styles, Interactive Learning Environments (2019) 1-21.

[41] R. Bartle, Hearts, clubs, diamonds, spades: Players who suit MUDs, Journal of MUD research 1 (1) (1996) 19.

[42] S. A. Kocadere, Çağlar, Gamification from player type perspective: A case study, Journal of Educational Technology \& Society 21 (3) (2018) $12-22$.

[43] W. O. dos Santos, I. I. Bittencourt, J. Vassileva, Gamification Design to Tailor Gamified Educational Systems Based on Gamer Types, in: SBGames, 2018.

[44] J. Hamari, J. Koivisto, Measuring flow in gamification: Dispositional flow scale-2, Computers in Human Behavior 40 (2014) 133-143.

[45] S. Hallifax, A. Serna, J.-C. Marty, G. Lavoue, E. Lavoue, Factors to Consider for Tailored Gamification, in: Proceedings of the Annual Symposium on Computer-Human Interaction in Play, CHI PLAY '19, ACM, New York, NY, USA, 2019, pp. 559-572.

[46] A. Mora, G. F. Tondello, L. E. Nacke, J. Arnedo-Moreno, Effect of personalized gameful design on student engagement, in: IEEE Global Engineering Education Conference (EDUCON), 2018, pp. 1925-1933. 
[47] W. Oliveira, A. Toda, P. Toledo, L. Shi, J. Vassileva, I. I. Bittencourt, S. Isotani, Does Tailoring Gamified Educational Systems Matter? The Impact on Students' Flow Experience, in: Proceedings of the 53rd Hawaii International Conference on System Sciences, 2020.

[48] R. J. Vallerand, L. G. Pelletier, M. R. Blais, N. M. Briere, C. Senecal, E. F. Vallieres, The Academic Motivation Scale: A measure of intrinsic, extrinsic, and amotivation in education, Educational and psychological measurement 52 (4) (1992).

[49] L. G. Pelletier, R. J. Vallerand, I. Green-Demers, N. M. Brière, M. R. Blais, Loisirs et santé mentale: les relations entre la motivation pour la pratique des loisirs et le bien-être psychologique., Canadian Journal of Behavioural Science/Revue canadienne des sciences du comportement 27 (2) (1995) 140.

[50] A. Field, J. Miles, Z. Field, Discovering statistics using R, Sage publications, 2012.

[51] A. B. Costello, J. Osborne, Best practices in exploratory factor analysis: Four recommendations for getting the most from your analysis, Practical assessment, research, and evaluation 10 (1) (2005) 7.

[52] J. C. Hayton, D. G. Allen, V. Scarpello, Factor retention decisions in exploratory factor analysis: A tutorial on parallel analysis, Organizational research methods 7 (2) (2004) 191-205.

[53] L. R. Fabrigar, D. T. Wegener, R. C. MacCallum, E. J. Strahan, Evaluating the use of exploratory factor analysis in psychological research., Psychological methods 4 (3) (1999) 272.

[54] L. Lee, S. Petter, D. Fayard, S. Robinson, On the use of partial least squares path modeling in accounting research, International Journal of Accounting Information Systems 12 (4) (2011) 305-328.

[55] M. R. Lepper, D. Greene, R. E. Nisbett, Undermining children's intrinsic interest with extrinsic reward: A test of the" overjustification" hypothesis., Journal of Personality and social Psychology 28 (1) (1973) 129. 
[56] E. L. Deci, R. Koestner, R. M. Ryan, Extrinsic rewards and intrinsic motivation in education: Reconsidered once again, Review of educational research 71 (1) (2001) 1-27.

[57] A. Stott, C. Neustaedter, Analysis of gamification in education, Surrey, BC, Canada 8 (2013) 36.

[58] D. Dicheva, C. Dichev, G. Agre, G. Angelova, Gamification in education: A systematic mapping study., Journal of Educational Technology \& Society 18 (3) (2015).

[59] S. HALLIFAX, A. Serna, J.-C. Marty, E. Lavoué, Dynamic gamification adaptation framework based on engagement detection through learning analytics, gamLA@LAK21 (Apr. 2021).

URL https://hal.archives-ouvertes.fr/hal-03196746 


\section{About the authors}

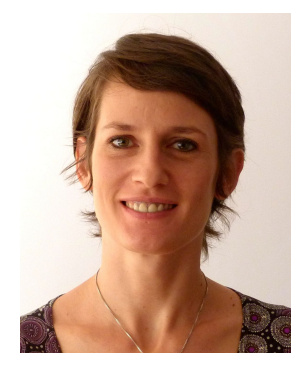

Élise Lavoué is an Associate Professor in Computer Science at the iaelyon School of Management, University Jean Moulin Lyon 3, and the head of the Situated Interaction, Collaboration, Adaptation, and Learning research group (SICAL) of the LIRIS laboratory. Her research interests include motivational techniques, tailored gamification, learners' engagement, and learning analytics dashboards, in the fields of TEL, CSCL, and HCI. She has (co)authored over 90 publications. She served as organizing chair for EC-TEL 2016, program committee co-chair for EC-TEL 2017, and general co-chair for CSCL 2019. She is SIG chair at EA-TEL, and a member of the EC-TEL Steering Committee.

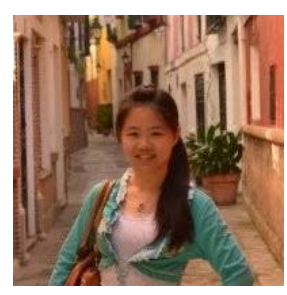

Qinjie Ju was awarded her PhD in 2019 from École Centrale de Lyon in the LIRIS computer science laboratory. Her main research interest is Humancomputer Interaction. Her $\mathrm{PhD}$ focused on gestural interaction for assisted music reading using fixed-gaze head movement, known as the EyeMusic system. More recently, she also worked on analyzing trace-based interaction for analysis of learners' engagement. 


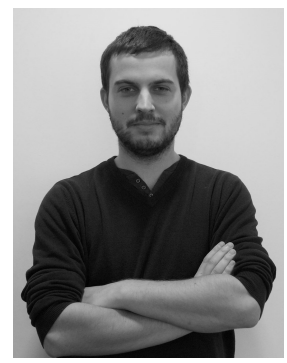

Stuart Hallifax was awarded his $\mathrm{PhD}$ from the University of Lyon 3 Jean Moulin in the LIRIS computer science laboratory. His main interests are Gamification, user behavior modeling, and user profiling. His $\mathrm{PhD}$ project focused on Adaptive Gamification of Computer-Based Pedagogical Resources.

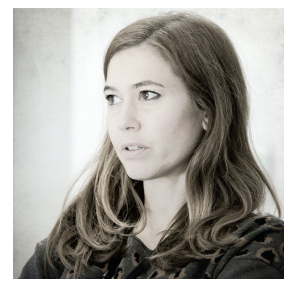

Audrey Serna is an Associate Professor in computer science at INSA de Lyon. Her research lies at the confluence of computer science (HumanComputer Interaction) and cognitive science (Cognitive Modeling/User Modeling) and focuses on designing adaptive, engaging, and supportive interactive systems. More specifically, she focuses on the design of novel interactive systems capable of adapting to the context of use, becoming increasingly variable at runtime and unforeseeable at design time. Her research mainly focuses on two complementary aspects: user modeling (human activity modeling, and analysis of users' engagement), and interaction with users (design of motivational affordances). 